\title{
Philology as a Systematic Method of Documentation and its Application to the Study of Constança Capdeville's Musical Works
}

\begin{abstract}
The advent of new music technologies has led to a rapid growth of the audio preservation field. Music is not independent of its medium, and this growing loss of independence calls for a transdisciplinary approach with a strong technological component. Musical compositions created in the second half of the twentieth century exist in the intersection of technology, embodiment, and sound and thus widen the importance of aspects such as human and non-human interactions. This article will reflect on these issues and how they reframe (digital) philology methods through the works of Constança Capdeville. The works by this seminal artist are accompanied by an extensive archive that includes scores, recorded sounds, video and images, among other documents. It will be demonstrated how a reflexive approach to digital philology can bring concealed archival stories to light while fostering new meanings on what it means to preserve our sound heritage. Resource optimisation is one of the reasons methods of traditional philology are particularly useful for the preservation of contemporary musical heritage. Today the amount of information is excessive, and categorisation and organisation are complex. Most information exists in the form of digitised documents, that comply with certain requirements traditionally applied to secular paper registers while assessing qualities such as reliability and authenticity. Traditional philology approaches framed around these qualities are insufficient for born-digital documents. A reflexive approach to digital philology, focussed both on the creation of digital resources (from digitalisation to cataloguing) and the criticism of digital sources, as a solution the digital treatment of documents is proposed. A comparative approach will be used to understand in what ways digital and analogue media intertwine in the forms we think, reflect, historicise, and preserve our sound archives, and how we make visible aspects that were previously concealed through new forms of categorising and digitising documents.
\end{abstract}

KEYWORDS: digital philology, audio preservation, documentation, contemporary Portuguese music, Constança Capdeville

\section{Introduction}

The theory and praxis of editing music almost exclusively focuses on written text. Music philology is closely bound to the concept of music notation, in which a composer records musical thought, and performance, where music is brought to life, thereby inevitably interpreted. 
In electro-acoustic music for recording media, on the other hand, the notion of an interpretative performance vanishes and the very concept of composition for magnetic tape seems to imply the music's reproducibility, thereby turning the concert performance into a mere repetition or, re-presentation of the piece (Straebel, 2009, p. 1).

This idea opens the way to a reconstruction of works that have come down to us in a purely aural or mixed paper-audio form (Benedictis, 2011, p. 1) showing the importance for the critical editions of music that include recording media. Traditional philology methods hold relevance to the preservation of contemporary musical heritage given their capacity to optimize resources. However, the type of materials produced and constituting the musical works from the second half of the twentieth century onwards usually reach beyond more traditional ideas of 'text', the fundamental object of philological studies. The inclusion in these works of media that do not fit into the more common conception of 'text', specifically the utilisation of fixed sounds in media, images, interactive systems, performance or other means, led philological studies to widen their borders. In addition, the information available about many musical works has now reached an excessive level of production and hence the need for its systematization and organization. For these documents to be readable and interpreted by future generations, they must comply with certain requirements that have previously been applied to secular paper records and inscribed in questions around reliability and authenticity. However, traditional philological approaches which, as a matter of principle, ensure reliable access to documents are insufficient for musical works when their documents are not confined to a single type or family. For example, they are not only paper documents or not merely digital documents, or they do not just feature images or sounds, regardless of the media, but are rather mutually incorporated. As stated by Bruno Bossis,

[...] it is essential to know how to read a document in order to understand it as an essential component of the research. A schematic transcription and the resolution of abbreviations and variable names, routines and sub-routines are indispensable. The composer or musical assistant can collaborate with the musicologist, but the pioneers of computer music will not always be available to analysts. Thus it is essential that sources are collected, documented and made accessible (Bossis, 2006, p. 110).

Yet according to the author,

[p]hilologists first examine documents in their physical reality, studying and evaluating the support media. They study the linguistic aspect and, finally, the literary aspect of the documents. Although etymologically, philology is concerned only with grammar and the exegesis and criticism of texts, it does more generally refer to everything which aids in the restoration and illustration of the past. In the same way, the musicologist must first of all determine the language of the music, which is sometimes very difficult considering the abundance of musical styles of the past century (Bossis, 2006, p. 110).

He maintains that the musicologist must also reconstruct the source's history, situating and precisely describing it. Generally speaking, a true scholarly apparatus is indispensable (Bossis, 2006, p. 110).

Thus, the diversity of documents contained in hybrid musical works requires the methods and practices of philology to be adjusted to this new paradigm. 
Digital philology, for example, which I shall explore below, focuses on two key aspects of digital documentary heritage: firstly, the creation of digital resources (from digitisation to the cataloguing of documents); then, secondly, on criticism of the currently available digital sources in the search for new solutions for handling such digital documents through an analogy with the treating of texts that is the object of traditional philology.

The authors Marc Leman and Joren Six quote James Turner, who in his book entitled Philology. The forgotten origins of the modern «humanities» (2014), claims that '[t]raditionally, philology was defined as the 'love of learning' about cultural heritage and it implied a focus on critical editions and comparisons of important texts that define our history and cultural heritage' (Leman \& Six, 2018, p. 309). The history of philology described by Turner is based on two facts: 1) the clear writing of the history of the Western humanities; and 2) the idea that philology is extended to other disciplines of modernism such as archaeology, history, linguistics, literary criticism, anthropology, and others. These disciplines share the common idea that historical lineage is essential to the understanding, considering that notions, texts, paintings, institutions, artifacts and languages are products of history shaped by their historical context (Turner, 2014, pp. 382-383). Turner does not present a final conclusion, but points out the various moments in history when it was difficult to deal with the excessive amount of information, with the selection of that information and, also, with the investigation of the techniques and technologies applied to the organization of such information (Turner, 2014, p. 383). A very succinct way of saying what philology is based on in Turner's considerations can be to consider it as a science that studies the history of languages and literature, its structure, its interrelations and its influence on culture of people. Philology is, in effect, a discipline that arose in antiquity and with a long-standing tradition regarding the study of historical textual sources. However, in the context of this work, I'm interested in the recent developments of this discipline, applicable not only to the arts and music in general, but in particular to Constança Capdeville's ${ }^{1}$ music.

\section{Text, philology and musical works}

In the article The Return to Philology (1995), Ivo de Castro seeks to demonstrate that philology is not reduced to the role of classifying the syntactic and lexical structures of a text. According to the author, it is important to consider 'the enormous variety of knowledge and expertise that philology can use to improve the text, from the recovery of the hidden part of the palimpsest to the detection of variants between two copies of the same edition' (Castro, 1995, p. 1). Castro borrows the title of a well-known article by Paul de Man, The Return to Philology, but which in itself is more directed towards the theory and teaching of literature.

\footnotetext{
${ }^{1}$ Constança Capdeville (1937-1992) - composer, pianist and percussionist, and the greatest representative of the music theatre in Portugal.
} 
The researcher Ana Paula Banza describes how philologists rescue works and retrieve their texts, contributing to the understanding of the period in which they were written and subsequently helping to uncover certain aspects of the sociopolitical, cultural, literary and linguistic history of a society. In this sense, the key philologist duty involves preserving cultural heritage, placing its work at the service of the society and the other communities (Banza, 2017, p. 17). Ivo de Castro states that the task of the philologist is to prepare a copy of a particular text in the form of a critical edition: removing the errors introduced in the course of the textual transmission and maintaining the traits that are presumably of the author's origin (Castro, 1995, p. 5). Therefore, and very briefly, in establishing a text, the author clings to two words: transcription and rectification, that is, the method of editing a text. This is the analogy envisioned to be established in the study of Constança Capdeville's music theatre works. However, as these include different documents (texts), such as scores, recorded sounds, video, images, texts, movement, scenic elements, among others, their study implies a need to authenticate and establish them within a single text able to aggregate the relationships between the various documents (texts), while considering both the transcription and the rectification, and this requires new approaches, since one is dealing with different types of 'texts' (see Figure 1).

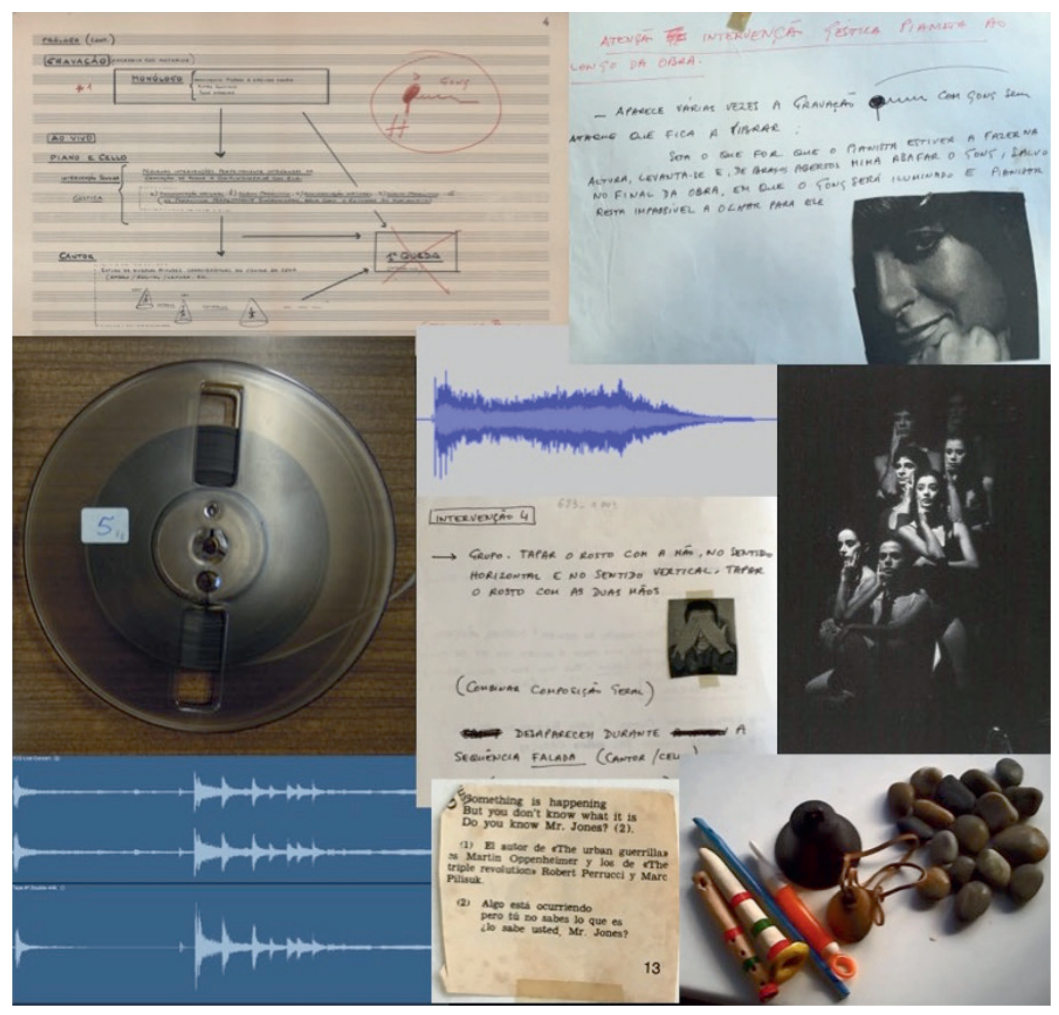

Figure 1. An example of the type of documents included in Capdeville's collection 
The study of these 'texts', as implicit in the discipline of philology, involves their authentication and integration in a coherent system, which incorporates the relationships between a variety of 'texts', taking into account the transcription of non-text documents, namely for new digital formats, and the articulation and systematization of these 'texts' to approach the author's original intention. The task of deciphering the original, identifying the error, understanding the conjecture, choosing the symbols, as endorsed by the philological methodology, entails risks that are directly linked to the interpretation of such documents. The knowledge of a document depends on the awareness of the philologist, or the musicologist (making a parallel with our specific case), about the various documents or 'texts' that s/he will analyse, and that interpretation can be somehow subjective.

In regard to the philologist's task, Castro argues that it reveals only a fragment of the text creation process $(1995$, p. 6). Still on the task of traditional philology, Laura Zattra recognizes that the main goal is to reconstruct the original form of the text as closely as possible to the author's intention. However, philology is currently concerned in searching the developments and modifications of this text, in order to outline it at every moment of its cultural history, seeking to understand issues of its mobility, instability and relationship with culture (Zattra, 2006).

According to Ana Paula Banza, in the 21st century, the materials (and consequently the various types of texts) become more easily accessible, allowing not only viewing them, as they were migrating to digital formats, but also establishing relationships between them through new information technology (IT) means. However, although technological evolution simplifies the visualization of the texts and the establishment of their relationships, it does not solve all problems. For example, if one of the texts is incomplete (whether it is a text itself or a score, a sound or a video), it is impossible to recover something that simply does not exist and, therefore, is considered lost. However, considering that the role of the philologist is to think of the text as a cultural product of an era, a product of a writing system, a product of a linguistic system (Banza, 2017, p. 19), the gap between the original text and the edited text will certainly be minimized.

In the musical context the same difficulties are found, but there are also some advantages. Comparing the type of documents that one can find in the context of musicology, certain 'texts' still need to be recognized as documentary sources. One refers in particular to the recorded sound. The scores, like any text in paper format, can be digitized and displayed on a computer screen. In the case of the 'texts' as the scores, the parallelism with traditional philology is evident (Bressan, 2018, p. 251). However, the sound recorded on a specific medium, which did not exist before the implementation of recording technology, becomes a new object within the scope of traditional philology, and the validation of a sound recorded as a source, comparable to a text, becomes essential. Nevertheless, any conversion, particularly in the case of a recorded sound, can raise problems, since it can interfere with the reality and quality of the original sound. Therefore, also the study of this type of 'text', the recorded sound, after its digitization, requires new approaches. Thus, while comparing a 'text' (as a recorded sound) with another 
'text' (literary), the differences between both are clear. Sound is linked to our auditory perception, being a very specific 'text', and for that reason it is important to determine its position in the new frame of reference imposed by the growing progress of digital formats.

\section{Recent trends in philology: on three approaches}

This article discusses three approaches that incorporate philological methods of documentation applied to the preservation of recordings or musical works, all of relevance to our study.

\subsection{Digital Philology}

The relationships between the real object (audio documents) and the digital object (the digitization of such object) have been approached through a new field of interdisciplinary research denominated 'Digital Philology'. According to the musicologist Federica Bressan, the field of philology, in a traditional sense, has generally agreed methods for reconstructing texts. Thus, the author is attentive to the way that digital technology influences the work of the philologist or the connection between philology and computer science within the audio document preservation context. In preparing the work materials, one should thus pay attention to issues such as the photographs taken of the audio carriers; the transcription and codification of the corpora texts; and the digitization of these recordings. Bressan draws particular attention to the importance of strictly applying philological principles to the preservation of audio documents so that the copy of this specific 'text', consisting of the audio document, becomes as reliable as possible.

In the perspective outlined above, this new field arises to answer questions that concern the relationship between the real object ${ }^{2}$ and the digital object, ${ }^{3}$ which is between the audio document ${ }^{4}$ and its digitization. In the article Hermeneutic Implication of Cultural Encoding. A Reflection on Audio Recordings and Interactive Installation Art, Bressan et al. (2017) continue this idea by reflecting on the emergence of this new field within the scope of philology as a discipline. The authors are once again concerned about the preservation of audio documents. The idea is that audio documents are used as sources or references in the study of musical works, specifically in the context of contemporary music. In the case of Constança Capdeville's music theatre works, whose

\footnotetext{
${ }^{2}$ A book, sheet music, namely physical documents in paper or audio format (e.g. magnetic tape, disc, CD, mini-disc, computer memory), which are now considered as sources, as it happens with paper documents.

${ }^{3}$ The digitization of paper documents, audio recordings, and so forth, therefore a document regardless of its format transformed into a digital object.

${ }^{4} \mathrm{An}$ audio document corresponds to the sound itself, the sound content, but this sound cannot be dissociated from its carrier (magnetic tape, vinyl record or an MP3, Wave format), nor from its contextual information.
} 
recordings on magnetic tape were used as an integral part of the works, these can benefit from this new philological approach. The application of philology and computer science methods to the preservation of audiovisual documents helps towards their preservation. Furthermore, this work will be completed with rigor and reliability as in traditional philology, rendering the audio documents as credible and useful sources while studying this type of repertoire. However, Capdeville's creations include other documents or 'texts' which are likewise relevant to the works themselves, requiring an articulation between all the elements in order to understand the entire work. It is, therefore, important to have a more comprehensive overview regarding other philological approaches, not limited to the textual ones, audio or video documents.

\subsection{Philology of Author}

The preservation, restoration and critical editing of music currently represent concerns to musicologists dedicated to the study of the various categories of contemporary music. In the article The critical editing of computer music (2006), Laura Zattra argues that the musicological study of electroacoustic music should incorporate an understanding of the sources so as to rethink the musicological approach in light of whatever might be considered as a source of electroacoustic music. According to the author, philologists focus their activities on the systematic analysis of the sources while exploring all of the existing documentation in emphasizing how the work of philologists is dynamic and developing over time and hence only ever inconclusive. Zattra maintains that including electronic sources into the study of music can benefit from the presence of the author or composer as their presence and documentation enables us to reach out to more sources as they are themselves the real testimonies of their work (2006, p. 1). The musicologist resorts to the notion of the 'Philology of Author' to demonstrate her statements. In the different categories of electroacoustic music, such witness sources might include: 1 ) an audio source (magnetic tape, CD, etc.); 2) a data storage device containing the digital data and algorithms; 3) printed digital scores; 4) traditional scores as in the case of mixed music; 5) sketches/ outlines by the author; 6) articles dedicated to the piece; 7) mental texts (the composer's memory, which aids in documenting musical thought). The analysis and comparison of these sources, which are often heterogeneous, then allows us to verify the history of a musical work and to understand its variants and transformations. In methodological terms, Zattra proposes that in a critical edition of a musical work including audio sources, the musicologist considers five fundamental procedures: a) to provide information about the musical work and its history, explaining the main criteria and problems; b) to critique the sources, listing the audio sources, digital scores, mental texts and witnesses; $c$ ) to describe accurately all sources; d) to make systematic collation ${ }^{5}$ between the various sources;

\footnotetext{
${ }^{5}$ In textual criticism it means comparing the various available versions of the same text, in order to reconstruct and evaluate its tradition or the various phases of its composition.
} 
e) to reconstitute the text (restoring its history, innovations and changes during its transmission and reception), this being the ultimate goal of the Author's Philology (Zattra, 2006, p. 3). Also with regard to rendering a critical edition of a work musical, Angelo Orcalli refers that 'the components of a musical work on an audiovisual medium are a kind of hologram of the multiple diffractions of compositional thought' (2017, p. 38). By this statement, the author means that preservation must constitute a return to life of the work at the time of its presentation in a concert. Arbo summarizes some of the methodological principles established by Orcalli, based on the different approaches to the preservation and restoration of audio documents, but somehow considers both the author's intention and the return to the musical performance, approaching the methodology defined by Zattra (2006, p. 3) while: safeguarding the documentary unit; understanding the type of document, comparing the different sources; considering the context (through the study of the characteristics of historical sound storage and distribution systems, aiming to recreate an audio document similar to what was heard by the viewers/listeners at the time of its transmission); reconstructing the documents in order to recreate the sound tissue, portraying the author's intention as much as possible; considering the field of aesthetics in order to make the recording more functional, both in terms of the listening and of the current technological standards (Arbo, 2018, p. 304).

Zattra also draws attention to the need of establishing collaborations between musicologists and audio engineers in order to find new forms of analysis, through the combination of both skills, allowing the analysis of documents related to the work, but also the analysis of the own work. For example, in Audio matching for the philological analysis of electroacoustic music (2007), Zattra collaborated with Nicola Orio in the design of a tool originally programmed in MATLAB, which would make it possible to compare two versions of the same work, facilitating its analysis. This survey arises in order to provide the musicologist with useful tools for the philological comparison of two different versions of the same work, having been directly applied to the study of John Chowning's Stria. ${ }^{6}$ Orio and Zattra report that this process reinforces the auditory analysis based on sonogram and spectrogram visualization tools.

Moreover, Orio and Zattra consider that the 'Philology of Author' extensively explores the revision activity carried out by the composer, in the light of his/her aesthetic and personal considerations, as well as his/her cultural and biographical context, arguing that it restores creative processes. Therefore, tools such as those mentioned above, can be useful in allowing comparisons of two versions of the same work, as happened with Stria by Chowning, or to compare audio records of interpretations, in different concerts, of any work. For example, when studying the collection of Teresa Rampazzi,7 Zattra found out that the composer would make various copies of audio recordings of the different versions of her works without, however, properly documenting this information (writing only

\footnotetext{
${ }^{6}$ For more information about this survey, see also Zattra (2007).

${ }^{7}$ Teresa Rampazzi 1914-2001 - Italian pianist and composer, pioneer in the field of electronic music in Italy and the first Italian woman to produce and promote this musical genre (Zattra, 2003, p. 11).
} 
the title of the works in audio recordings). Hence, the analysis of these audio records by comparing the different audio sources was very useful to identify the different recordings (Orio \& Zattra, 2007, p. 157). Thus, the analysis and comparison of audio records allows one to verify the history of a musical work and understand its variants and transformations. Constança Capdeville's music theatre works also include heterogeneous sources (different audio recordings of the same work) and, therefore, the application of this methodology, which is based on the comparison and analysis of different sources, proves to be useful for the study of her works, also leading to their understanding.

\subsection{Ontological reflections on the preservation and restoration of musical artifacts}

Alessandro Arbo considers that a recording can either document a work (or a musical event) or constitute it. The author argues that musical works are considered as artifacts that operate aesthetically and correspondingly strives to perceive how the artifact was conceived, considering this approach appropriate to preserving and restoring musical works. Arbo states that recordings may assume different roles: a document, a recording of a musical event or even itself constituting a musical moment. With regard to the preservation and restoration of musical works, the author recommends two important tasks: firstly, a preservation work that proposes to safeguard the integrity of the original trace and the means; being in agreement with the proposal of 'Digital Philology' as advocated by Bressan et al. (2017), aiming to transfer the content of the analogue (original) audio or video carriers to digital formats; secondly, an understanding of the document in the form of a musical work, creating the conditions for an effective activation of its functioning. Still regarding the preservation and restoration of audio recordings, Arbo makes considerations similar to the work developed at Mirage ${ }^{8}$ which in regard to the practice of remediation ${ }^{9}$ of audio documents defends the view that

the implications of the stochastic paradigm in the documentary and specifically philologicalmusical setting are relevant and inevitable for any musicologist who needs to be equipped accordingly: in order to adjust the source criticism to the characteristics of the medium it is necessary to forfeit seeing authenticity as a quality of the document per se, viewing it rather as a property of its production or re-mediation process. Musical work itself is a process whose witnesses (recordings) are its 'states', defined by the constraints of the technology of the time and by the models of sound (Orcalli, 2017, p. 29).

${ }^{8}$ Laboratory based at the University of Udine, under the direction of Professor Angelo Orcalli (founder and scientific coordinator) and Luca Cossettini (Audio Archive coordinator). Available at http://mirage.uniud.it (accessed: 09.10.2020).

${ }^{9}$ Remediation is the action of transferring acoustic information from one medium to another one and, currently, the only solution to the problem of degradation of the analogue carriers in order to avoid an irreversible loss of information (Bressan \& Canazza, 2013, p. 1). 
In fact, the ontological issues of musical works are also an aspect to take into account in their preservation and restoration. And that is not only the audio documents that are part of the work itself, but also those that result from the performance. For example, Arbo admits that recordings may help to document a work, and considers that musical works are artifacts operating aesthetically, thus he seeks to understand how these artifacts are produced. The nature of musical works is also considered since they are works of art that are closely linked to the previous lines and can be filed in any type of document (audio or video). Some musical works include scores, recordings or other documents, and therefore require special care that is often devalued. Many archivists and conservators are unaware of the ontological relevance of the works they deal with and should take this issue into consideration in order to understand what types of documents constitute these works.

Constança Capdeville's collection is housed at the National Library of Portugal (BNP), but an archivist who is unaware of the particularities of such musical works will find many difficulties in understanding the type of documents and their organization. Ideally, the archivist should have specific knowledge about the creative process. Capdeville's music theatre works contain audio recordings that are occasionally incorporated into the work itself or can help to document it (e.g. audio or video recordings of performances representing a musical event, but also include scores, scripts, sketches, among others). Also, many of Capdeville's works do not include conventional scores and, when they do exist, are not written in a conventional way (see also Magalhães \& Pires, 2019, p. 97). Consequently, even to attain simple information on how to determine the duration of the work is difficult and is only possible from the audio or video recordings of the performance itself or, for instance, through the knowledge of the people who worked directly with Capdeville, knowing exactly what she intended, since the composer paid special attention to the aspects of gesture, movement, rendering the testimony of these people essential. Thus, the documentation of such works, in fact, should be made by a musicologist specialized in this particular range. Accordingly, the idea defended by Arbo, when he states that the nature of each document must be understood, serves the study of Capdeville's musical works.

Thus, the philology applied to music embraces now the problem of the preservation and the obsolescence of the technology, of both analogue and even digital formats, due to rapid technological evolution, and to the urgent need to keep these documents accessible. This is also one of the problems that Arbo discusses, the obsolescence of the formats, which can be damaged, lost or become unreadable. The author gives as an example the case of electronic music, rock, jazz, since often these musical categories are not written on scores (whether in conventional notation, tablature, chords or other), and the non-existence of the recording of such events risks losing track of them (Arbo, 2018, p. 306). The same happens with part of Capdeville's music theatre works, as these are still not yet properly preserved and documented. There are no video recordings of the performances, and since these included the scenic component it is still difficult to understand such an aspect through the documentation available. Besides the 
loss of some audio recordings that are incorporated in the performances, it is still unknown whether some of the works have already been partially or totally lost, and if it is still possible to recover them.

\section{The application of existing philology methods to the musical works of Constança Capdeville}

In terms of my own experiences when studying Capdeville's musical works, recourse to the performance's recordings (both audio and video, whenever existing) was crucial to approaching her musical works. Of course, there are certainly some flaws, for example technological obsolescence, which indeed already afflicts some of Capdeville's tapes, but there are also the difficulties in grasping the articulation between the various layers of information contained in these documents, including distinguishing between the sound sources: the acoustic and electronic sounds. The surviving documents (scores, scripts, slides, images, sketches, and so on, as depicted in the figure) need to be systematized. A digital format is the only system capable of grouping all of these documents for their subsequent articulation; as if rendering the intangible material. Unfortunately, not all of the musical works of Constança Capdeville include video susceptible both to assisting in documenting the respective creative process and to supporting our understanding of aspects such as gesture, movement or others. For example, certain sections of the performance might be missing, or the sound does not attain a good enough quality standard and thereby hamper our understanding of the interactions between the various facets of these works.

The above-mentioned approaches facilitate the study of Constança Capdeville's music-theatre works, above all due to their applicability, but still some of the methods used are inadequate. Digital Philology, as explained above, is relevant, because Capdeville's works involve real objects (audio carriers, scores, paper, images) and digital objects (the digitization of audio carriers, scores or the other documents). However, it is worth mentioning that in the case of Capdeville's collection, the documents are highly diversified, are not restricted to audio or video carriers, and most of them have not been yet digitized properly in current formats. After the digitization of the collection, such documents will need to be observed from other aspects of philology. The Philology of the Author, advocated by Laura Zattra, is also pertinent, but it cannot be directly applied to Capdeville's work, since she is no longer available to elaborate upon her thoughts, although there is still the chance to count on the memory of some of the interpreters who collaborated with her.

The problems that Zattra faced with Rampazzi's collection are very similar to those found on Capdeville's tape collection. In the Capdeville collection one finds some audio recordings of the performances of her works, as it succeeds in Mise-en-Requiem (1979); or, in some cases, the performance rehearsal record appears on the tape, as it occur in Libera me $(1977,1979,1981)$, with choreography by Vasco Wellenkamp, music by Constança Capdeville, and stage design and costumes by Emília Nadal (Nogueira, 2018, p. 5). In both cases, 
a comparison with other sources would be desirable, since it would make it possible to draw instructions, namely from changes that could arise in the rehearsals or in the performances. Nevertheless, once again, Capdeville's works are not limited to audio recordings, as they include scores, scripts, slides, images, in short, a myriad of different types of documents, consequently the tool that Zattra and Orio applied to the study of John Chowning's Stria is also revealed to be insufficient.

Despite Capdeville's particular case, where one cannot count on the presence of the composer, one can still resort to the documentation left by her, which can be studied and compared, and also to the testimonies of the various actors that participated in the composer's performances. This collaboration also provides us with new sources and relevant information about the creative process, helping to produce new documentation. Arbo's perspective, in turn, when suggesting that recordings of performances can contribute to reconstructing a musical work, is a useful conception for the study of Capdeville's musical works, since it is necessary to resort to the recordings of the performances (both audio and video), when they exist, to systematize the musical work.

The various approaches in the field of philology of music described above prove to be particularly relevant, given the heterogeneity of the sources included in Capdeville music-theatre works (from scores to audio or video recordings, scripts, sketches, and other supplementary documents). The aforementioned proposals also contribute to the restoration of the work as close as possible to the original 'text', bringing us closer to what would be the author's intention, taking into account the context in which the musical work was produced and presented, and it is in this sense that I seek to guide this proposal.

Thus, for this study, the philological method adopted for the treatment and systematization of the various documents in Capdeville's musical works proposes:

- to identify the various sources (scores, scripts, audio or video recordings, sketches, images, testimonies, and so forth), as suggested by Zattra in the methodology set out above;

- to interview the artists who collaborated or participated in the performances, because, as in the 'mental texts', above-mentioned by Zattra, these artists witnessed aspects of the creative process and can, therefore, provide new sources and help to understand some facts of the productions. From these sources and reports, therefore, new documentation is produced;

- to retrieve documents, audio or others, and migrate their content to current formats; paper documents and images will be treated (when necessary) and digitized, as well as audio documents will be transferred to new formats in a digital environment, taking into account the assumptions of 'Digital Philology' (which aims to apply the principles of traditional philology to the preservation of audio documents, so that the copy of this specific 'text', or audio document, is as reliable as possible), as suggested by Bressan et al (2017) and Arbo (2018); 
- to make a detailed study of the audio recording(s), identifying each sound in order to understand the inputs of that recording used throughout the performance (regarding the methodological approach, the procedures and techniques of the systematic musicology). Because one deals with recordings and these require a lot of precision regarding their analysis, it is important to understand the durations of the various sounds in order to establish consistency in the systematization of the work;

- to describe and compare the various sources (or documents); analyzing the score (where it exists) while listening to the recordings used during the performance (as in the context of electroacoustic music) and the recordings (audio or video) of the live performances (where they exist), using whenever necessary the various scripts (since the information is scattered and incongruous), or other supplementary documentation (sketches or extra instructions from the composer or the performers), especially to help in building a general structure of the work;

- to perform the replacement of the text, taking into account its historical context, innovations and changes over time (using other writings on the work and press review).

The last procedure presents itself as the final result in all the aforementioned approaches (both 'Digital Philology', as well as the 'Philology of the Author' or others), namely with regard to the critical edition of musical works, as it aims to approach the author's original intention, which is, in essence, the goal of philology.

\section{Conclusion}

Interweaving the approaches outlined above holds particular relevance given the sheer heterogeneity of the sources involved in Constança Capdeville's musical works (from scores to audio recordings, video recordings, scripts, sketches as well as other additional documents). The proposals presented here serve not only to contribute towards reconstructing the original form of the text but also potentially revealing hidden practices behind the creative process within the scope of bringing us as close as possible to the author's original intentions through assuming the contexts in which these musical works were produced and presented.

To conclude, our interpretations of such documents are extremely important in conjunction with the ways in which we opt to convey our interpretations. In this kind of research, making clear the methods deployed and assessing our own research footprints hold equal relevance. This spans not only the ways in which documentation is used but also the respective processes to which it is subject. In methodological terms, the most important factor is to document and clearly explain both what is being done and how it is being done. Historical knowledge 
on the type of repertoire is fundamental to enabling an understanding of Constança Capdeville's musical works. Nevertheless, building a narrative from the existing documents, integrating the archive, the repertoire and the musical practices all still remain crucial-so that the works are understandable as a whole as well as restoring contemporary music to the modern history panorama.

\title{
Translated by Filipa Magalhães
}

\author{
I would like to thank to Simon Curtis for his generous willingness to \\ carefully revising the English of this text, which greatly contributed \\ to improving its readability.
}

\section{References}

Arbo, A. (2018). From the document to the work: Ontological reflections on the preservation and restoration of musical artefacts. Journal of New Music Research, 47(4): 300-308. doi: 10.1080/09298215.2018.148643.

Banza, A. P. (2017). Da antiga à nova Filologia: práticas de edição de textos modernos. In R. Trachsler, F. Duval, L. Leonardi (éd.), Actes du XXVIIe Congrès international de linguistique et de philologie romanes, (Nancy, 15-20 juillet 2013). Section 13: Philologie textuelle et éditoriale. ATILF.

Benedictis, A. I. de (2011). Materiali parziali o strutture fungibili? Nuove prospettive filologichesu 'Honeyrêves', 'Don Perlimplin'e 'SerenataIV' di Bruno Maderna. Il Saggiatore Musicale. Rivista semestrale di musiologia, anno XVIII, 1-2: 139-171.

Bossis, B. (2006). The Analysis of Electroacoustic Music: from sources to invariants. Organised Sound, 11(2): 101-112. doi:10.1017/S135577180600135X.

Bressan F. (2018). A Philological Approach to Sound Preservation. In L. Levenberg, T. Neilson, D. Rheams (Eds.), Research Methods for the Digital Humanities, (pp. 243-261). Palgrave Macmillan, Cham. doi: 10.1007/978-3-319-96713-4_14.

Bressan, F. (2017). Philology in the preservation of audio documents. Customized versus readymade approaches. In A. Tandon, A. Slomska, J. Opoku-Boateng, \& D. Abbazia (Eds.), Unlocking Sound and Image Heritage: Selected Readings fromt the 2015 SOIMA Conference, (pp. 69-74). International Centre for the Study of the Preservation and Restoration of Cultural Property (ICCROM). doi: 10.18146/soima2015.

Bressan, F., \& Canazza, S. (2013). A Systemic Approach to the Preservation of Audio Documents: Methodology and Software Tools. Journal of Electrical and Computer Engineering, 2013: 1-22.

Castro, I. de (1995). O Retorno à Filologia. In C. de Cunha \& P. R Dias Pereira (Eds.), Miscelânea de Estudos Linguísticos, Filológicos e Literários in Memoriam Celso Cunha, (pp. 511-520).

Leman, M., \& Joren S. (2018): Beyond documentation - The digital philology of interaction heritage. Journal of New Music Research, 47(4): 309-320 doi: 10.1080/09298215.2018.1479428.

Magalhães, F., \& Pires, I. (2019). Recovering Music-Theatre Works Involving Electronic Elements: The case of Molly Bloom and FE...DE...RI...CO.... Organised Sound, 24(1), 96-108. doi:10.1017/ S1355771819000098.

Nogueira, A. (2018). Que Futuro para o Património Musical Contemporâneo Nacional? Documentar para Preservar, (Doctoral dissertation, Universidade NOVA de Lisboa, Portugal). Retrieved from http://hdl.handle.net/10362/58912. 
Orcalli, A. (2017). Recorded music: from the ethics of preservation to the critical editing. In L. Cossettini \& A. Orcalli (Eds.), Sounds, Voices and Codes from the Twentieth Century, (pp. 3-81). University of Udine: Department of Languages and Literatures, Communication, Education and Society.

Orio, N., \& Zattra, L. (2007). Audio Matching for the Philological analysis of electroacoustic music. Proceedings of the International Computer Music Conference, 2007: 157-164.

Straebel, V. (2009). The 'Project for Magnetic Tape' (1952/53): Challenging the Idea of a Critical Edition of Historic Music for Recording Media. Paper at Music: Notation and Sound, joint conference of the International Association of Music Libraries (IAML) and the International Musicological Society (IMS), Amsterdam.

Turner, J. (2014). Philology: The forgotten origins of the modern humanities. Princeton: Princeton University Press.

Zattra, L. (2006). The critical editing of computer music. Paper at Electroacoustic Music Studies Conference (EMSo6) - Theme: Terminology and Translation, Beijing, China. Retrieved: http:// www.ems-network.org/IMG/EMS2006 - LZattra.pdf.

Zattra, L. (2007). The Assembling of 'Stria' by John Chowning: A Philological Investigation. Computer Music Journal, 31(3): 38-64. 